\title{
Picosecond photoresponse in Y-Ba-Cu-O ultrathin films
}

\author{
L. Shi, ${ }^{*}$ G. L. Huang, C. Lehane, D. Kim, and H. S. Kwok \\ New York State Institute on Superconductivity and Department of Electrical and Computer Engineering, \\ State University of New York at Buffalo, Bonner Hall, Amherst, New York 14260 \\ J. Swiatkiewicz, G. C. Xu, and P. N. Prasad \\ Department of Chemistry, State University of New York at Buffalo, Bonner Hall, Amherst, New York 14260
}

(Received 30 April 1993)

\begin{abstract}
We have applied a double-bridge voltage-correlation technique to study the nonequilibrium superconducting properties in high- $T_{c}$ superconductors. Using $\mathrm{Y}-\mathrm{Ba}-\mathrm{Cu}-\mathrm{O}$ thin films of thickness less than 15 $\mathrm{nm}$, we have observed voltage pulses as fast as $40 \mathrm{ps}$. The temporal photoresponse signal was found to depend strongly on the sample's thickness. A systematic trend in the superconductivity decay time as a function of film thickness was obtained. The phonon-trapping effect has been verified to be the major limiting mechanism in the quasiparticle (QP) relaxation process. The role of electron-phonon coupling in the QP decay process has also been studied with 500-fs and 40-ps laser pulses.
\end{abstract}

\section{INTRODUCTION}

Nonequilibrium superconductivity can be obtained by exposing superconductors to various external excitation sources such as photons, phonons, current injection, and microwaves. Testardi recognized that photoexcitation generates quasiparticles (QPs) in superconductors without introducing a branch mixing effect. ${ }^{1}$ Since then, the generation of transient voltage pulses in superconductors by laser irradiation has been studied extensively. ${ }^{2,3}$ Parker and Williams used laser illumination to study the reduction of the order parameter $\Delta$ and changes in the QP's chemical potential $\mu_{p} \cdot{ }^{4}$ Narayanamurti et al. applied the optical injection technique to superconducting thin films..$^{5}$ They were able to separate the phonon and QP signals by detecting them with a bolometer and a junction in a time-of-flight measurement. Chi et al. proposed an optical autocorrelation scheme using the threshold effect of superconducting-normal transition. ${ }^{6}$ QP recombination time of 500 ps was observed in a thickness variable bridge. Later, they also studied $\mathrm{Pb}$ junctions using a 40-ps laser system. ${ }^{3}$

Since the discovery of high- $T_{c}$ superconductors (HTSC), Y-Bu-Cu-O (YBCO) in particular, there has been an increasing interest in studying their nonequilibrium superconducting properties. ${ }^{7-16}$ Much attention has been focused on their optical response because of (1) their possible applications as photodetectors or other optoelectronic devices, (2) the possibility of unveiling the fundamental physical processes involved with the high$T_{c}$ superconductivity, (3) the success of applying optical techniques to low- $T_{c}$ superconductors, and (4) the short coherence-length nature of the high- $T_{c}$ oxides. Bluzer has used a transient photoimpedance response method to study the QP relaxation in HTSC and Nb thin films. ${ }^{13,16}$
The observed voltage signals were detected by an ultrafast Hypres oscilloscope and were interpreted as the kinetic inductance change due to optically generated QPs. They have observed 70-ps QP recombination time in a 30-nm-thick YBCO thin film. ${ }^{16}$ This method overcomes the disadvantage of the conventional junction techniques, ${ }^{17}$ but relies on a very fast oscilloscope which has a temporal resolution of 20 ps. By taking advantage of the temporal resolution of ultrashort laser pulses, we have developed a double-bridge voltage-correlation (DVC) technique. ${ }^{11,15}$ The temporal resolution of DVC is limited only by the laser-pulse duration and transit-time effects. Voltage pulses faster than 40 ps have been observed in microstrip lines fabricated on 10-nm-thick YBCO films. This technique not only provides the necessary temporal resolution for studying the dynamics of these nonequilibrium phenomena but also eliminates the need of an ultrafast oscilloscope. ${ }^{12,13}$

In this paper, we report further results of our doublebridge experiment on YBCO ultrathin films with the emphasis on measuring their ultimate photoresponse time and understanding its origin. Voltage pulses as fast as 40 ps have been generated in YBCO thin films of thickness less than $15 \mathrm{~nm}$. For the first time, the superconductivity decay time as a function of film thickness was systematically studied. The phonon-trapping effect has been verified to be the major limiting mechanism in the $Q P$ relaxation process. Using laser pulses of durations of $500 \mathrm{fs}$ and $40 \mathrm{ps}$, we have also studied the role of electronphonon coupling in the QP decay process.

\section{EXPERIMENTAL TECHNIQUE AND SAMPLE PREPARATIONS}

The conventional beam splitter Michelson interferometer arrangement, as shown in Fig. 1, was employed in the 


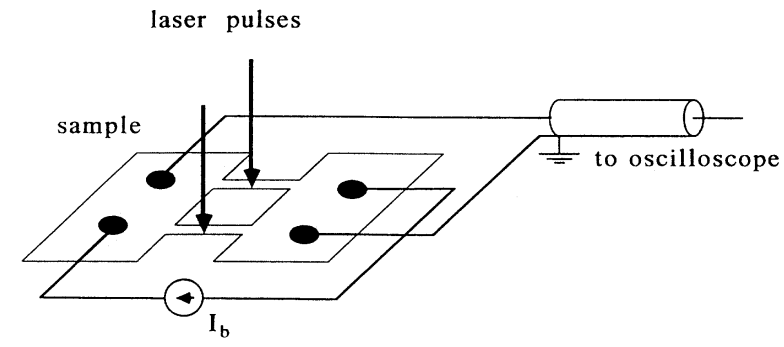

FIG. 1. Schematic diagram of the double-bridge experiment. The two laser pulses are derived from the same laser with a controlled time delay.

two-beam voltage-correlation experiment. The laser pulse was split into two equal channels and time delayed. A fine translation stage was used to provide the time resolution needed in the optical delay. A single $10-\mathrm{cm}$ focal length lens was applied to direct both beams onto the microbridges through a standard dewar. The purpose of this lens was to provide bending and focusing of the laser beams to ensure uniform illumination. The spot size of the laser beam on the sample was about $0.25-\mathrm{mm}$ radius, which was less than the spacing between the two bridges. Neutral density filters placed in the optical paths could be used to vary the laser intensity.

When the laser pulses are time delayed and the bridges are unbalanced, the current redistributes to minimize the total resistance. A resistance $R(t)$ can be used to describe the voltage state of the bridges. The timedependent voltage across the bridges can therefore be given as

$$
V(t, \tau)=I_{b}\{R(t) R(t-\tau) /[R(t)+R(t-\tau)]\},
$$

where $I_{b}$ is the total dc bias current and $\tau$ is the time delay between the two pulses. Taking into account the contributions of the scope limiting bandwidth $\Delta B(\sim 1 / 2 \delta)$ and trigger jitters $\xi$, the displayed signal $V_{\text {osc }}^{*}(t, \tau)$ is related to the input signal $V(t, \tau)$ by ${ }^{13}$

$$
V_{\mathrm{osc}}^{*}(t, \tau)=(1 / N) \sum 1 /(2 \delta) \int_{t-\delta-\xi}^{t+\delta-\xi} V\left(t^{\prime}, \tau\right) d t^{\prime},
$$

where $N$ is the number of pulses averaged. By measuring the peak signal on the oscilloscope as a function of time delay $\tau$, we can obtain a correlationlike curve of the voltage pulse. A simple biexponential expression

$$
R(t)=\exp \left(-t / \tau_{1}\right)+A \exp \left(-t / \tau_{2}\right)
$$

is used to fit the experimental data.

Optically thin YBCO films are used mostly in this work to minimize the phonon-trapping effect. ${ }^{3,18,19}$ Thermal transport within the light absorption depth can also be ignored. Such effects have to be considered for the optically thick films $>100 \mathrm{~nm}$. Uniform distributions of QPs and hot phonons can therefore be assumed and greatly simplifies the data analysis. These thin films are deposited on $\mathrm{MgO}$ (100) substrates using the method of pulsed laser deposition (PLD). Figure 2 shows typical $R-T$ curves of YBCO thin films with thickness. The $T_{c}$ of the 7.5-, 10-, and 100-nm films are 70, 78, and $90 \mathrm{~K}$, re-

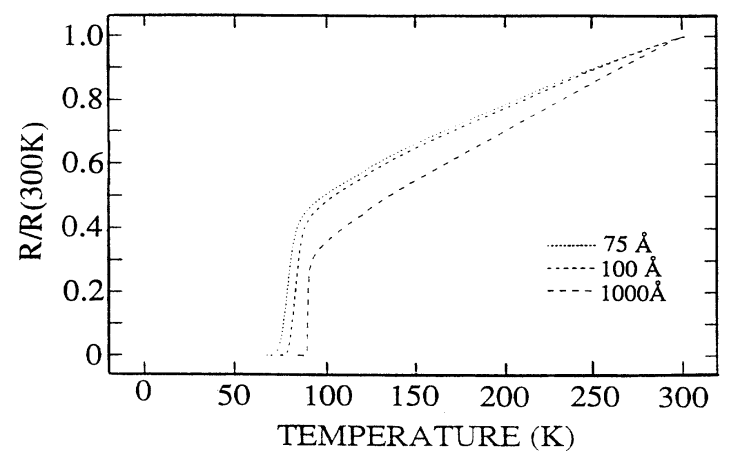

FIG. 2. Typical $R-T$ curves of unpatterned YBCO thin films on $\mathrm{MgO}$ (100) substrate deposited by PLD.

spectively. Stripline patterns are then prepared by direct laser writing, or standard photolithography. For most experiments discussed in the following, double bridges of $50 \mu \mathrm{m}$ (width) $\times 500 \mu \mathrm{m}$ (length) lines are used. Since the superconductivity properties of the microbridge are very sensitive to the film uniformity as the film thickness approaches $10 \mathrm{~nm}$, the separation between the bridges is minimized to be as small as possible $(<500 \mu \mathrm{m})$.

Two laser sources are used to provide the necessary temporal resolution: 500-fs laser pulses derived from a multistage amplified synchronously pumped dye laser and 40-ps laser pulses generated from an actively and passively mode-locked Nd:YAG laser oscillator. In the experiment, YBCO ultrathin films were irradiated by the 500-fs or 40-ps laser pulses. A serious problem for both laser systems is the shot-to-shot fluctuation of the optical laser intensity. This large fluctuation, combined with the low laser repetition rate, explains the larger data fluctuations presented below.

\section{THEORETICAL BACKGROUND}

In general, the relaxation times of quasiparticles (QP's) and phonons for a nonequilibrium superconductor depend upon the strength of the laser irradiation. As incident photons disturb the occupation of the QP and phonon states, four basic processes in the superconductors are likely to happen, and they are characterized by different time parameters: ${ }^{19,20}$ (1) A QP scatters with the emission or absorption of a phonon $\left(\tau_{s}\right)$; (2) Two QP's recombines to form a Cooper pair with the emission of a phonon $\left(\tau_{R}\right)$; (3) Creation of two QP's from the pair condensate through the absorption of a phonon $\left(\tau_{B}\right)$; (4) High-energy phonons escape from the film to the substrate $\left(\tau_{\text {es }}\right)$. To fully describe the time dependence of the measured voltage signal, a calculation of the coupled Rothwarf and Taylor rate equations of QP's, Cooper pairs, hot phonons, and acoustic phonons is necessary. ${ }^{21}$ However, we can understand these dynamic processes qualitatively as following. ${ }^{3,13}$ In the superconducting state, optical photons with energies of the order of $2 \mathrm{eV}$ will cast the Cooper pairs and QP's greatly out of equilibrium, since the YBCO superconducting energy gap $2 \Delta$ is of the order of $30-40 \mathrm{meV}$. These photons will break Cooper pairs and create QP's at energies much larger 
than the gap energy $\Delta$. Successive interaction between these high-energy QP's and the Cooper-pair condensate divides the excess energy by generating more QP's. At the initial stage, the electrons decay very rapidly via electron-electron interaction (cascading process) to thermalize toward the energy gap $2 \Delta(T)$. The electronelectron process slows down as the hot QP's lose their excess energies, and eventually electron-phonon interaction takes over. ${ }^{3,13}$ Large amounts of $2 \Delta$ optical phonons are generated during the QP thermalization process. Whether these hot phonons remain in the optically illuminated region to break more Cooper pairs or escape into the "cold" substrate depends on the thermal coupling between the film and the substrate. These two processes of phonon pair breaking and phonon escaping are governed by time $\tau_{B}$ and $\tau_{\text {es }}$, respectively. There exist two extreme cases: ${ }^{19,21}$ (1) fast phonon escape, $\tau_{\mathrm{es}}<<\tau_{B}$, the measured QP lifetime is approximately given by the intrinsic QP lifetime $\tau_{R}$. (2) slow phonon escape, $\tau_{\text {es }} \gg \tau_{B}$, and the measured QP lifetime is enhanced by a factor of $\left(1+\tau_{\text {es }} / \tau_{B}\right)$, the phonon-trapping factor. These two cases can be distinguished by measuring the $\mathrm{QP}$ lifetime in films of different thickness, since the size of this phonontrapping effect is a strong function of the film thickness $d .^{22}$ This precisely is the purpose of this experiment.

\section{EXPERIMENTAL RESULTS AND DISCUSSIONS}

Voltage pulses faster than 100 ps have been routinely generated in the superconducting state of ultrathin YBCO samples with thickness of less than $30 \mathrm{~nm}$ by the DVC technique. Figure 3 shows the fast voltage pulses measured on two 10-nm-thick thin films using 500-fs and 40-ps laser pulses. To facilitate the comparison, two

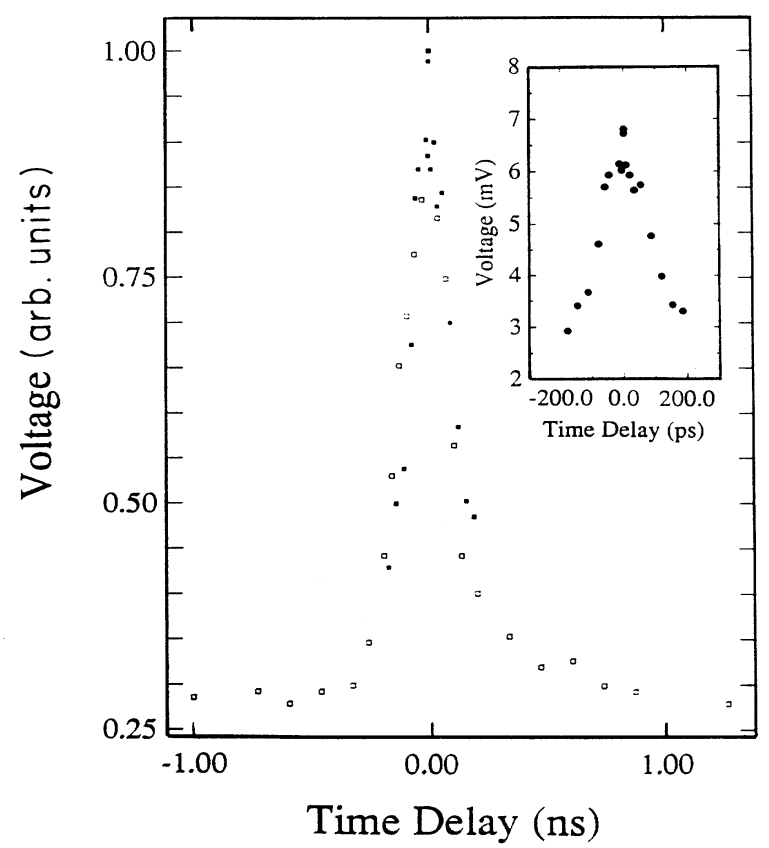

FIG. 3. QP recombination time of two 10-nm-thick YBCO thin films. They were obtained by using 40-ps and 500-fs laser pulses. Inset shows the 500-fs result on an absolute scale. traces were normalized to their peak values. The zero point was determined by a standard autocorrelation measurement. These voltage pulses were numerically fitted by the biexponential expression described above. The fast component can be fitted very well by a time constant of $40 \mathrm{ps}$, while the slow one has a characteristic time of $\sim 2 \mathrm{~ns}$. It is evident that voltage pulses as fast as $40 \mathrm{ps}$ were clearly demonstrated, and their decay times did not depend very sensitively on the excitation laser-pulse durations. The inset shows the 500-fs trace in an absolute scale. These two samples were measured at temperatures around $25 \mathrm{~K}$. The dc currents were adjusted such that they were biased at $\frac{1}{3}-\frac{1}{2}$ of the films' $I_{c}$ and the samples remained in the superconducting state. It is worth pointing out that the YBCO thin films irradiated by $500-\mathrm{fs}$ laser pulses usually gave rise to much lower thermal background component compared to that by 40-ps pulses. As a matter of fact, we have observed a "clean" voltage pulse of $\sim 150$-ps duration in a $35-\mathrm{nm}$ thin film using 500-fs laser pulses. The trailing portions on both sides were well below our measurement sensitivity of 500 $\mu \mathrm{V}$. It is obvious that the voltage pulse shapes can be quite different for different amount of heat deposited into the films. It is thus possible that the difference of using 500-fs laser and 40-ps laser is due to the effective temperature rise in the film or the difference of biasing conditions.

Our interpretation for the above result is that the fast component is due to QP generation and decay. Due to the hot-phonon-trapping effect, the QP recombination time is not simply $\sim\left(1 / N_{q p}\right)$ but is enhanced by the trapping factor $\left(1+\tau_{\text {es }} / \tau_{B}\right)$. Therefore in a voltage measurement, the decay process is determined by the hot-phonon escape time from the superconducting film. The slow component is related to cooling and heat diffusion which is essentially the escape of all phonons, mostly acoustic phonons from the film. It is instructive to estimate the expected voltage pulse duration by hot-phonon trapping. As analyzed in Ref. 3, the QP relaxation time is on the order of the phonon escape time $\tau_{\text {es }}$, which is given by

$$
\tau_{\mathrm{es}}=4 d / \eta v_{s}
$$

where $d$ is the film thickness, $\eta$ is the transmissivity of the hot phonon across the boundary, and $v_{s}$ is the speed of phonons. Using the value of $v_{s}\left(=5 \times 10^{5} \mathrm{~cm} / \mathrm{s}\right)$ and assuming $\eta=0.2, \tau_{\text {es }}$ can be estimated to be $40 \mathrm{ps}$ for the 10-nm-thick film. Of course, detailed knowledge of $\eta$ is important in this estimation. Furthermore, as the film gets thinner, a simple calculation such as Eq. (3) breaks down.

We have found that the QP decay time is very sensitive to the operating conditions such as ambient temperature, bias current, and laser fluence. The most intriguing parameter is the bias current. Despite our effort to reproduce the samples deposition and patterning process, we have observed some inconsistent results in the relaxation time from several samples. In one experiment, when a 25-nm-thick film was biased very close to $T_{c}$, the voltage pulse displayed a 40-ps fast decay near $\tau=0$, followed by a slow decay. When we lowered the bias current, the fast 
component became longer and had a time constant of 100 ps. We performed a similar experiment in a $15-\mathrm{nm}$-thick film. The film was either in the superconducting state, transition state, or normal state by varying the bias current across the bridges. Instead of observing pulse shortening, the voltage pulses were actually much broader when biased near $T_{c}$. The correlation of bias current to QP decay time is not very clear at this stage. We speculate that it possibly depends on the quality of the film and its structure (see below), and this may also apply to many other reported observations as well. ${ }^{12-14}$ Figures 4(a) and 4(b) show the temperature and bias current dependence of the fast voltage response in a 15nm-thick film. The voltage signals were clearly nonthermal nature in origin, since the fast voltage pulses could be still observed at temperatures below $T_{c}$ where $d R / d T$ is zero, similar to Ref. 7. The bias dependence in (a) is similar to that reported in Ref. 10.

Besides the temperature and bias current dependence of these fast signals, another important characteristics is the film thickness dependence of the QP relaxation time. ${ }^{22,24}$ The QP decay time vs thickness plot is summarized in Fig. 5. Only the short decay times measured around $25 \mathrm{~K}$ were plotted in the graph. The large error bar for the 25-nm-thick film was the result of two successive measurements at different thermal cycles. As noted before, the experimentally measured $\mathrm{QP}$ recombination time is longer than the intrinsic time $\tau_{R}$ by a phonontrapping factor due to repeated pair-breaking and recombination processes. The size of this factor depends on the ratio of the phonon mean free path against pair-breaking $\Lambda$ and the film thickness $d$ as well as the phonon transmission coefficients $\eta \cdot{ }^{18,19,22}$ The thickness dependence of the QP recombination time we obtained is quite consistent with the calculation in Ref. 22, where three
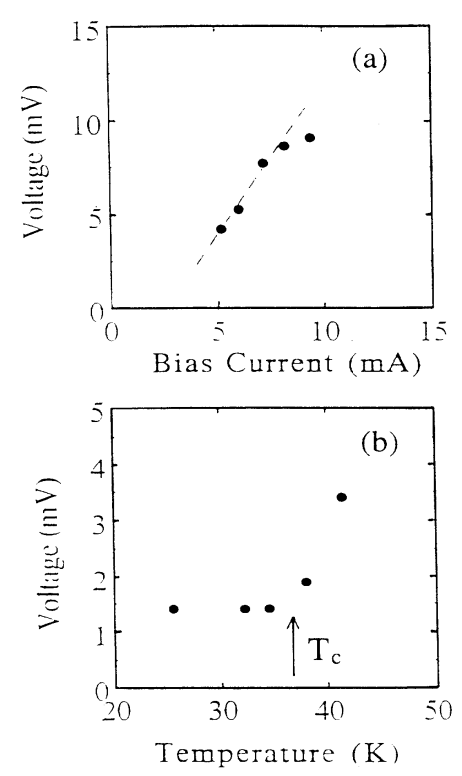

FIG. 4. (a) Bias current dependence of the voltage signal at $25 \mathrm{~K}$. The dotted line is a guide to the eye. (b) Temperature dependence at bias current of $3 \mathrm{~mA}, T_{c}$ is about $37 \mathrm{~K}$.

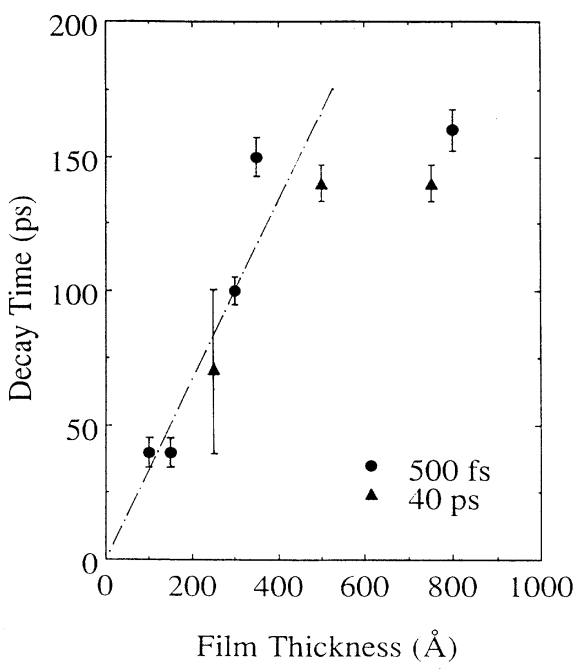

FIG. 5. QP decay time as a function of the film thickness. The 500-fs and 40-ps results were represented by the circles and triangles. The dashed line is calculated from Eq. (3).

distinct regions were found by solving the RothwarfTaylor equations. The phonon-trapping affects the QP lifetime mostly in the linear region, which covers most of the film thickness we have studied. The dashed line in Fig. 5 was calculated from Eq. (3) by assuming $\eta=0.24$, it fits this linear dependence quite well. This clearly suggests that the phonon escaping process is the limiting mechanism for QP relaxation in our measurement. The QP decay time can be estimated from the phonon escaping time $\tau_{\text {es }}$. It is also clear that comparisons of calculated and measured quasiparticle decay times are capable of yielding information about the phonon-trapping effects and thermal boundary conditions in this region. On the other hand, the volume loss of phonons becomes important and cannot be neglected for the thicker films, which effectively shortens the QP recombination time. ${ }^{18,19,22}$ This well explains why the QP decay time starts to deviate from the values predicted by Eq. (3) in the thicker film region in Fig. 5.

It is also interesting to estimate the ultimate intrinsic QP recombination time. For the thin films of $d<<\Lambda$ and if phonons are reflected specular by the substrate, $\tau_{\text {eff }}^{-1}=\tau_{R 1}^{-1}\left(1-\cos \theta_{c l}\right)+\tau_{R t}^{-1}\left(1-\cos \theta_{c t}\right){ }^{22}$ Using the published phonon velocities and densities for both YBCO and $\mathrm{MgO},{ }^{25,26}$ i.e., for YBCO: $V_{1}=5.0 \times 10^{5} \mathrm{~cm} / \mathrm{s}$, $V_{t}=2.9 \times 10^{5} \mathrm{~cm} / \mathrm{s}, \quad \rho=6.35 \mathrm{~g} / \mathrm{cm}^{3}$; for $\mathrm{MgO}$ : $V_{1}=9.7 \times 10^{5} \mathrm{~cm} / \mathrm{s}, V_{t}=6.1 \times 10^{5} \mathrm{~cm} / \mathrm{s}, \rho=3.58 \mathrm{~g} / \mathrm{cm}^{3}$, critical angles for the total reflection of longitudinal and transverse waves $\theta_{\mathrm{cl}}$ and $\theta_{c t}$ can be calculated to be $31.03^{\circ}$ and $28.39^{\circ}$, and thus $\tau_{\text {eff }}^{-1}=\tau_{R 1}^{-1}(0.143)+\tau_{R t}^{-1}(0.120)$. If we only consider one phonon mode, and take $\tau_{\text {eff }}=40 \mathrm{ps}, \tau_{R t}$ (or $\tau_{R 1}$ ) can be estimated to be about $5 \mathrm{ps.} \mathrm{This} \mathrm{value} \mathrm{is}$ quite consistent with the $\mathrm{QP}$ recombination time obtained from femtosecond transient reflectivity measurements. ${ }^{27}$ It should be important to measure the QP decay time for even thinner films $(d<10 \mathrm{~nm})$. Since ultrathin YBCO films of a few unit-cell thick are now readily available, these experiments should be possible. Recently, 
Ghis et al. measured a 30-ps recovery time in a 35-nmthick film. ${ }^{28}$ Their result is in general agreement with our data.

Although Fig. 5 clearly displays a trend that strongly suggests a QP dynamics dominated by phonon trapping, we have observed a large variation in QP relaxation time from sample to sample. We have examined more than ten films $(d \approx 10-15 \mathrm{~nm})$, films with higher $T_{c}$ generally exhibit faster response time (or QP decay time). We speculate that there is a strong dependence of QP lifetime on the superconducting film structural properties, especially for ultrathin films. ${ }^{23}$ It is well known that ultrathin films are very different from that of thick films in terms of their properties of dimensionality and superconductivity. As discussed above, the QP decay times in these ultrathin films are essentially the phonon escaping time, which is very much decided by the film and substrate thermal coupling conditions. Therefore, it is not too surprising that the interface quality could affect the photoresponse time in the ultrathin films. More experiments are needed to verify this assumption.

\section{PHONON-RELATED PROCESSES}

\section{A. Thermal boundary resistance and heat transport time}

In most cases, the film temperature rise due to the incident radiation can be derived from a simple heat transfer model, where the thermal boundary resistance between the film and the substrate is neglected. ${ }^{10}$ However, it has been shown that the thermal boundary resistance plays a significant role during the heat transport in thin films as compared to the thermal resistance of the superconducting film in the thick films. ${ }^{29}$ Furthermore, a qualitative knowledge of the thermal boundary is crucial in distinguishing thermal or nonthermal mechanisms in the photoresponse experiment. ${ }^{30,31}$ Although Figs. 4(a) and 4 (b) have clearly indicated a nonthermal origin for our voltage signals, it is still worthwhile to estimate the effect of thermal boundary conditions on the heat transfer process. The thermal boundary resistance $R_{b d}$ is defined as the ratio of the temperature difference $\Delta T$ across the interface to the power per unit area $\dot{Q} / A$ flowing across it, i.e., $R_{b d}=\Delta T A / \dot{Q}$. At very low temperatures, the acoustic mismatch model has been very successful in explaining this boundary resistance, where $R_{b d} \propto T^{-3}$. But at higher temperatures, the phonon wavelengths are so short that the interface imperfections and irregularities have to be considered. In the case of YBCO thin films, the $T^{-3}$ law breaks down at high temperatures and $R_{b d}$ approaches a constant value of $\approx 0.8-1.4 \times 10^{-3}$ $\mathrm{K} \mathrm{cm}{ }^{2} / \mathrm{W}$ at $100 \mathrm{~K}^{30}$

We have numerically solved the coupled thermal diffusion equations using the method outlined in Ref. 31. Both variable thermophysical properties and contact resistance between the film and the $\mathrm{MgO}$ substrate were taken into account. In the calculation, we assumed that the acoustic mismatch model still holds at low temperature for the YBCO thin films. At a laser intensity of 100 $\mu \mathrm{J} / \mathrm{cm}^{2}$, the temperature rise inside the film is about $9 \mathrm{~K}$ for $500-\mathrm{fs}$ laser pulses. This relatively large temperature rise is the result of short laser-pulse duration and long thermal transit time $\tau_{t} \cdot{ }^{31}$ If the film temperature is considered lumped, $\tau_{t}$ can be estimated by ${ }^{30,31}$

$$
\tau_{t}=c_{f} \rho_{f} R_{b d} d,
$$

where $c_{f}, \rho_{f}$, and $d$ are the specific heat, density, and thickness for the YBCO film. Since the $R_{b d}$ values are not generally available at low temperatures, we use the high-temperature $(100 \mathrm{~K})$ value of $1 \times 10^{-3} \mathrm{~K} \mathrm{~cm} / \mathrm{W}$ for $R_{b d}$. For $d=10 \mathrm{~nm}, \tau_{t}$ can be estimated to be about 200 ps at $26 \mathrm{~K}$ (for comparison, $\tau_{t}$ is $1.6 \mathrm{~ns}$ at $50 \mathrm{~K}$ ). Interestingly, this gives the lowest bound of $\tau_{t}$, if we assume that the $T^{-3}$ law is still valid at temperatures below 30 $\mathrm{K}$. Clearly, $\tau_{t}$ is proportional to the film thickness $d$, and the thinner film will give rise to a faster heat transfer time $\tau_{t}$. However, our 40-ps result cannot be simply explained by this thermal transit time $\tau_{t}$.

\section{B. Electron-phonon coupling time}

Recently, Gershenzon et al. suggested that the electron-phonon relaxation time in YBCO is about $40 \mathrm{ps}$ at $4 \mathrm{~K}$, and decreasing as $1 / T$ with temperature. ${ }^{32}$ This implies that $\tau_{\text {ep }}$ is of $6.5 \mathrm{ps}$ at $25 \mathrm{~K}$. Ballentine et al. have observed a fast rising time in an optically thick film at temperature below $20 \mathrm{~K} .{ }^{33}$ They interpreted their result by the hot-electron transport effect. Since this $\tau_{\text {ep }}$ value lies between the laser-pulse durations we used in the experiment, it is interesting to examine the electronphonon coupling effect on the QP relaxation process.

When the laser-pulse duration is longer than the electron-phonon thermal relaxation time $\tau_{\text {ep }}$, obviously the hot QP's have enough time to establish a local thermal equilibrium with the lattice and the QP's and phonons have the same temperature. The effects of microscopic photon-electron, electron-electron, and electron-phonon interactions are insignificant, which is possibly the case of our 40-ps pulses. While for our 500fs laser pulses, which are shorter than the electronphonon thermal relaxation time $\tau_{\text {ep }}$ in the HTSC films, the electron-phonon interaction should become an important controlling process in the transfer of energy. Since the QP heat capacity is small, QP's can be heated to a very high temperature within a very short time. QP's and the phonons are no longer in local thermal equilibrium and have to be considered as two separate systems. ${ }^{34}$ Thermalization of the photoexcited QP's occurs via electron-electron and electron-phonon interactions. The electron-phonon thermal relaxation time $\tau_{\text {ep }}$ is inversely proportional to the electron-phonon coupling strength.

In superconductors, the QP and phonon transport processes, and the electron-phonon relaxation process remove energy from the hot QP's within the radiation penetration depth. Since our DVC method cannot detect the initial rise time of the voltage pulse, we cannot detect the hot-electron transport effect. This transport time for a 25-nm-thick film can be as short as $6.25 \mathrm{fs}$. However, as suggested in Ref. 3, the electron-phonon coupling would affect the QP decay process. After photoabsorption, the electron temperature can be much greater than the lattice temperature, i.e., $T_{e} \gg T_{\mathrm{ph}} \gg T_{s}$, we would 
expect an energy transfer from electrons to phonons through electron-phonon coupling as $C_{e}\left(T_{e}-T_{\mathrm{ph}}\right) / \tau_{\mathrm{ep}}$ and similarly, an energy transfer from phonons to the substrate through phonon escape as $C_{\mathrm{ph}}\left(T_{\mathrm{ph}}-T_{s}\right) / \tau_{\mathrm{es}}{ }^{35}$ These two interfering mechanisms should result in two decay time constant in our 500-fs photoresponse data and one component in the 40-ps data. However, besides the difference in the background component amplitude, there are no observable distinction between data of using 500-fs or 40-ps laser pulses. This suggests that either the electron-phonon coupling time is shorter than $500 \mathrm{fs}$ or longer than 40 ps at $25 \mathrm{~K}$ in these HTSC thin films. This is quite contrary to the results in Ref. 32, but quite consistent with the femtosecond pump-probe data. ${ }^{27}$ We believe that this can be further resolved by performing the photoresponse and femtosecond pump-probe experiment simultaneously on the superconducting microbridges.

\section{SUMMARY}

We have introduced a technique for measuring the QP decay time of fast electrical pulses in YBCO microstrip-

*Present address: Laboratory for Laser Energetic, and Department of Electrical Engineering, University of Rochester, Rochester, NY 14623.

${ }^{1}$ L. R. Testardi, Phys. Rev. B 4, 2189 (1971).

${ }^{2}$ I. Shuller and K. E. Gray, Phys. Rev. Lett. 36, 429 (1976).

${ }^{3}$ C. C. Chi, M. M. T. Loy, and D. C. Cronemeyer, Phys. Rev. B 23, 124 (1981).

${ }^{4}$ W. H. Parker and W. D. Williams, Phys. Rev. Lett. 29, 924 (1972).

${ }^{5}$ V. Narayanamurti, R. C. Dynes, P. Hu, H. Smith, and W. F. Brinkman, Phys. Rev. B 18, 6041 (1978).

${ }^{6}$ C. C. Chi, M. M. T. Loy, D. C. Cronemeyer, and M. L. Thewalt, IEEE Trans. Magn. MAG-17, 88 (1981).

${ }^{7}$ H. S. Kwok, J. P. Zheng, Q. Y. Ying, and R. Rao, Appl. Phys. Lett. 54, 2473 (1989).

${ }^{8}$ E. Zeldov, N. M. Amer, G. Koren, and A. Gupta, Phys. Rev. B 39, 9712 (1989).

${ }^{9}$ W. R. Donaldson, A. M. Kadin, P. H. Ballantine, and R. Sobelewski, Appl. Phys. Lett. 54, 2470 (1989).

${ }^{10}$ A. Frenkel, M. A. Saifi, T. Venkatesan, P. English, X. D. Wu, and A. Inam, J. Appl. Phys. 67, 3054 (1990).

${ }^{11}$ H. S. Kwok, L. Shi, J. P. Zheng, S. Y. Dong, Y. Pan, and P. N. Prasad, in On The Speed of Optically-Controlled Superconducting Devices, SPIE Proceedings on Progress in HighTemperature Superconducting Transistors and Other Devices, edited by R. Singh, J. Narayan, and D. T. Shaw (SPIE, Bellingham, WA, 1991), Vol. 1394, p. 196.

${ }^{12}$ M. Johnson, Phys. Rev. Lett. 67, 374 (1991); Appl. Phys. Lett. 59, 1371 (1991).

${ }^{13}$ N. Bluzer, J. Appl. Phys. 71, 1336 (1992); Phys. Rev. B 44, 10222 (1991).

${ }^{14}$ A. D. Semenov, G. N. Goltsman, I. G. Gogidze, A. V. Sergeev, P. T. Lang, and F. F. Renk, Appl. Phys. Lett. 60, 903 (1992).

${ }^{15}$ L. Shi, G. L. Huang, C. Lehane, J. P. Zheng, and H. S. Kwok, Appl. Phys. Lett. 61, 489 (1992).

${ }^{16}$ N. Bluzer, IEEE Trans. Appl. Supercond. 3, 2869 (1993).

${ }^{17}$ B. I. Miller and A. H. Dayem, Phys. Rev. Lett. 18, 1000 (1967).

${ }^{18}$ S. B. Kaplan, J. Low Temp. Phys. 37, 343 (1979). lines. It is based on the standard technique of autocorrelation in ultrafast spectroscopy. We have demonstrated the ability of generating and measuring voltage pulses faster than 40 ps. Systematic studies of temperature, current, and film thickness effects on these fast voltage pulses supported a QP mechanism. Phonon-trapping effect has been verified to be the major limiting factor on the observed superconductivity relaxation process. The transmissivity of the hot phonons was found to be about 0.24 at $25 \mathrm{~K}$. Laser pulses of 500-fs and 40-ps durations were used in the experiment, which has enabled us to study the role of electron-phonon coupling in QP decay process. Besides obvious technological applications in ultrafast electronics, these fast events occurring in the superconducting state offer new challenges to theoretical investigations.

\section{ACKNOWLEDGMENT}

This research was supported by NSF Grant No. ECS9017053.

${ }^{19}$ J. J. Chang and D. J. Scalapino, J. Low Temp. Phys. 31, 1 (1978).

${ }^{20}$ S. B. Kaplan, C. C. Chi, D. N. Langenberg, J. J. Chang, S. Jafarey, and D. J. Scalapino, Phys. Rev. B 14, 4854 (1976).

${ }^{21}$ A. Rothwarf and B. N. Taylor, Phys. Rev. Lett. 19, 27 (1967).

${ }^{22}$ W. Eisenmenger, K. Lassmann, H. J. Trumpp, and R. Krauss, Appl. Phys. 11, 307 (1976).

23J. L. Levine and S. Y. Hsieh, J. Low Temp. Phys. 20, 471 (1971).

${ }^{24}$ G. A. Sai-Halasz, C. C. Chi, A. Denenstein, and D. N. Langenberg, Phys. Rev. Lett. 33, 215 (1974).

${ }^{25}$ H. E. Fisher, S. K. Watson, and D. G. Cahill, Comments Condens. Matter Phys. 14, 65 (1988).

${ }^{26} \mathrm{G}$. Simmons and H. Wang, Single Crystal Elastic Constants and Calculated Aggregate Properties: A Handbook (M.I.T., Cambridge MA, 1971).

${ }^{27}$ S. G. Han, Z. V. Vardeny, K. S. Wong, O. G. Symko, and G. Koren, Phys. Rev. Lett. 65, 2708 (1990).

${ }^{28}$ A. Ghis, S. Pfister, J. C. Villegier, M. Nail, and J. P. Maneval, IEEE Trans. Appl. Supercond. 3, 2136 (1993).

${ }^{29}$ S. Zeuner, H. Lengfellner, J. Betz, K. F. Renk, and W. Prettl, Appl. Phys. Lett. 61, 973 (1992).

${ }^{30}$ M. Nahum, S. Verghese, P. L. Richards, and K. Char, Appl. Phys. Lett. 59, 2034 (1991).

${ }^{31}$ M. I. Flik, P. E. Phelan, and C. L. Tien, Cryogenics 80, 1118 (1990).

${ }^{32}$ E. M. Gershenzon, M. E. Gershenzon, G. N. Gol'tsman, B. S. Karasik, A. D. Semenov, and A. V. Sergeev, Pis'ma Zh. Eksp. Teor. Fiz. 46, 226 (1987) [JETP Lett. 46, 285 (1987)].

${ }^{33}$ P. H. Ballentine, A. M. Kadin, W. R. Donaldson, J. H. Scofield, and L. Bajuk, in Optically Triggered Switching of Superconducting $\mathrm{YBa}_{2} \mathrm{Cu}_{3} \mathrm{O}_{7}$ Thin Films, SPIE Proceedings on High $T_{c}$ Superconductivity: Thin Films and Applications, edited by Cheng-Chung Chi and R. Bruce van Dover (SPIE, Bellingham, WA., 1990), Vol. 1287, p. 134.

${ }^{34}$ T. Q. Qiu and C. L. Tien, Int. J. Heat Mass Transfer, 35, 719 (1992).

35J. P. Maneval, F. Chibane, and R. W. Bland, Appl. Phys. Lett. 61, 339 (1992). 